\title{
Erythropoietin-Stimulating Agent Hyporesponsiveness in Patients Living with Chronic Kidney Disease
}

\author{
Henry H.L. Wu ${ }^{a, b}$ Rajkumar Chinnadurai ${ }^{b, c}$ \\ aDepartment of Renal Medicine, Lancashire Teaching Hospitals NHS Foundation Trust, Preston, UK; ${ }^{b}$ Faculty of \\ Biology, Medicine and Health, The University of Manchester, Manchester, UK; ' Department of Renal Medicine, \\ Northern Care Alliance NHS Foundation Trust, Salford, UK
}

\section{Keywords}

Anemia · Erythropoietin-stimulating agent ·

Hyporesponsiveness · Chronic kidney disease

\begin{abstract}
Background: Erythropoietin-stimulating agent (ESA) hyporesponsiveness is commonly observed in patients with anemia secondary to chronic kidney disease (CKD). Because of its complexity, a global consensus on how we should define ESA hyporesponsiveness remains unavailable. The reported prevalence and demographic information on ESA hyporesponsiveness within the CKD population are variable with no consensus definition. Summary: ESA hyporesponsiveness is defined as having no increase in hemoglobin concentration from baseline after the first month of treatment on appropriate weight-based dosing. The important factors associated with ESA hyporesponsiveness include absolute or functional iron deficiency, inflammation, and uremia. Hepcidin has been demonstrated to play an important role in this process. Mineral bone disease secondary to CKD and non-iron malnutrition among other factors are also associated with ESA hyporesponsiveness. There is continued debate toward determining a gold-standard treatment pathway to manage ESA
\end{abstract}

karger@karger.com www.karger.com/kdd

Karger $\frac{1}{\%}$
(C) 2022 The Author(s)

Published by S. Karger AG, Basel

This is an Open Access article licensed under the Creative Common Attribution-NonCommercial-4.0 International License (CC BY-NC) (http://www.karger.com/Services/OpenAccessLicense), applicable to the online version of the article only. Usage and distribution for commercial purposes requires written permission. hyporesponsiveness. The development of hypoxia-inducing factor-stabilizers brings new insights and opportunities in the management of ESA hyporesponsiveness. Key Message: Management of ESA hyporesponsiveness involves a comprehensive multidisciplinary team approach to address its risk factors. The progression of basic and clinical research on identifying risk factors and management of ESA hyporesponsiveness brings greater hope on finding solutions to eventually tackling one of the most difficult problems in the topic of anemia in CKD.

(C) 2022 The Author(s)

Published by S. Karger AG, Basel

\section{Introduction}

Anemia is frequently observed in patients living with advanced chronic kidney disease (CKD) and associated with adverse outcomes [1]. Since approval for use by the US FDA in 1989, erythropoietin-stimulating agents (ESAs) represent an important therapeutic agent in anemia management for advanced CKD patients. USRDS 2020 Annual Data report highlighted more than $85 \%$ of hemodialysis (HD) patients received ESA treatment [2]. 
ESA hyporesponsiveness remains a difficult problem in clinical practice. Currently, there is no standardized global definition to define ESA hyporesponsiveness. Since no unified definition is determined, reported prevalence of ESA hyporesponsiveness varies according to the definition applied. ESA hyporesponsiveness portrays worsened prognosis [3]. Escalating ESA doses to achieve hemoglobin $(\mathrm{HgB})$ targets may elevate cardiovascular, thrombotic, and subsequently mortality risks [4]. The complex relationship between ESA dose and HgB levels is increasingly appreciated. Research continues to expand on the numerous factors which influence this dynamic and nonlinear relationship. Iron deficiency, inflammation, and the role of hepcidin in these processes is a recent topic of interest from the literature relating to ESA hyporesponsiveness $[5,6]$. Other factors such as uremia, CKDmineral bone disease (CKD-MBD), and non-iron malnutrition are also increasingly studied $[7,8]$.

Identifying factors and treating reversible causes should be initially pursued when managing ESA hyporesponsiveness [9]. Decision whether to continue ESA treatment hinges on symptom control of anemia, morbidity, quality of life status, and future opportunities of kidney transplantation [9]. The past decade has gone by with new innovations to manage anemia in CKD including achieving dialysis adequacy in HD patients through convective $\mathrm{HD}$, vitamin E-bonded dialysis membrane application, and other methods to improve membrane permeability [10-12]. Progressive development of hypoxia inducible factor (HIF) prolyl-hydroxylase inhibitors (HIF-stabilizers), now in phase III clinical trials, allows for endogenous production of erythropoietin [13-15]. Administered orally, cost-effective benefits of HIF-stabilizers to manage anemia in CKD are evident [16]. There are concerns regarding carcinogenic effects of HIF-stabilizers however, and it remains to be seen whether they are the optimal solution over long term [17].

In this review, we will recap the updated definitions and prevalence of ESA hyporesponsiveness in CKD and the surrounding debate. Recent evidence relating to clinical outcomes of ESA hyporesponsiveness will be reviewed. Our latest understanding of factors contributing to ESA hyporesponsiveness will be discussed, and mechanisms of these factors will be explored. We will evaluate pathways to treat anemia in CKD and how ESA hyporesponsiveness could be minimized. Based on current evidence, we aim to conject a practical approach to address ESA hyporesponsiveness in renal anemia management and identify remaining gaps in our knowledge base which may provide future avenues of research.

\section{Defining ESA Hyporesponsiveness in CKD}

There is no consensus definition for ESA hyporesponsiveness internationally. In the revised European Best Practice Guidelines (ERBG) 2004, evaluation of ESA hyporesponsiveness in CKD patients is recommended if there is increase in erythropoietin dose $\geq 25 \%$ to maintain the same $\mathrm{HgB}$ level or $<1 \mathrm{mg} / \mathrm{dL}$ gain in $\mathrm{HgB}$ after $2-4$ weeks [18]. The Kidney Disease Improving Global Outcomes (KDIGO) 2012 guidelines defined initial ESA hyporesponsiveness as having no increase in $\mathrm{HgB}$ concentration from baseline after the first month of treatment on appropriate weight-based dosing [19]. For CKD patients receiving consistent doses of ESA treatment initially, subsequent ESA hyporesponsiveness is defined as those requiring 2 instances of increased ESA doses up to $50 \%$ beyond the dose at which they had been stable in an effort to maintain similar $\mathrm{HgB}$ concentration levels [19]. The Kidney Disease Outcomes Quality Initiative/National Kidney Foundation and Kidney Health Australia-Caring for Australasians with Renal Impairment guidelines currently recommend KDIGO 2012 definition for ESA unresponsiveness [20,21]. Updated definitions of ESA hyporesponsiveness in select guidelines are summarized in Table 1 [18-23].

One quantitative measure of ESA hyporesponsiveness developed in the past 15 years is the ESA resistance index (ERI). ERI is based on a ratio between ESA dose per kilogram and $\mathrm{HgB}$ level based on weekly averages [24]. It is suggested to play a role in outcome prediction and ESA dosage guidance $[3,25]$. However, the validity of ERI remains controversial, and ERI is not yet advocated for use in the clinical setting by most guidelines [24].

\section{Prevalence of ESA Hyporesponsiveness in CKD}

National registry data reporting the prevalence of ESA hyporesponsiveness are currently unavailable. Anemia is expected to be more frequently observed in dialysis than in nondialysis CKD patients. In an observational study encompassing both $\mathrm{HD}$ and peritoneal dialysis patients, Bae et al. [26] divided ERI into tertiles to represent the degree of ESA responsiveness and noted $33 \%$ of $\mathrm{HD}$ or peritoneal dialysis patients were classified into the highest tertile, where the highest tertile represents patients with poorest ESA responsiveness. From other cohort studies primarily focusing in HD groups, ESA hyporesponsiveness varied between 5 and $20 \%$ in the HD population [27, 28]. Minutolo et al. [29] 
Table 1. Selected definitions of ESA hyporesponsiveness in patients living with CKD

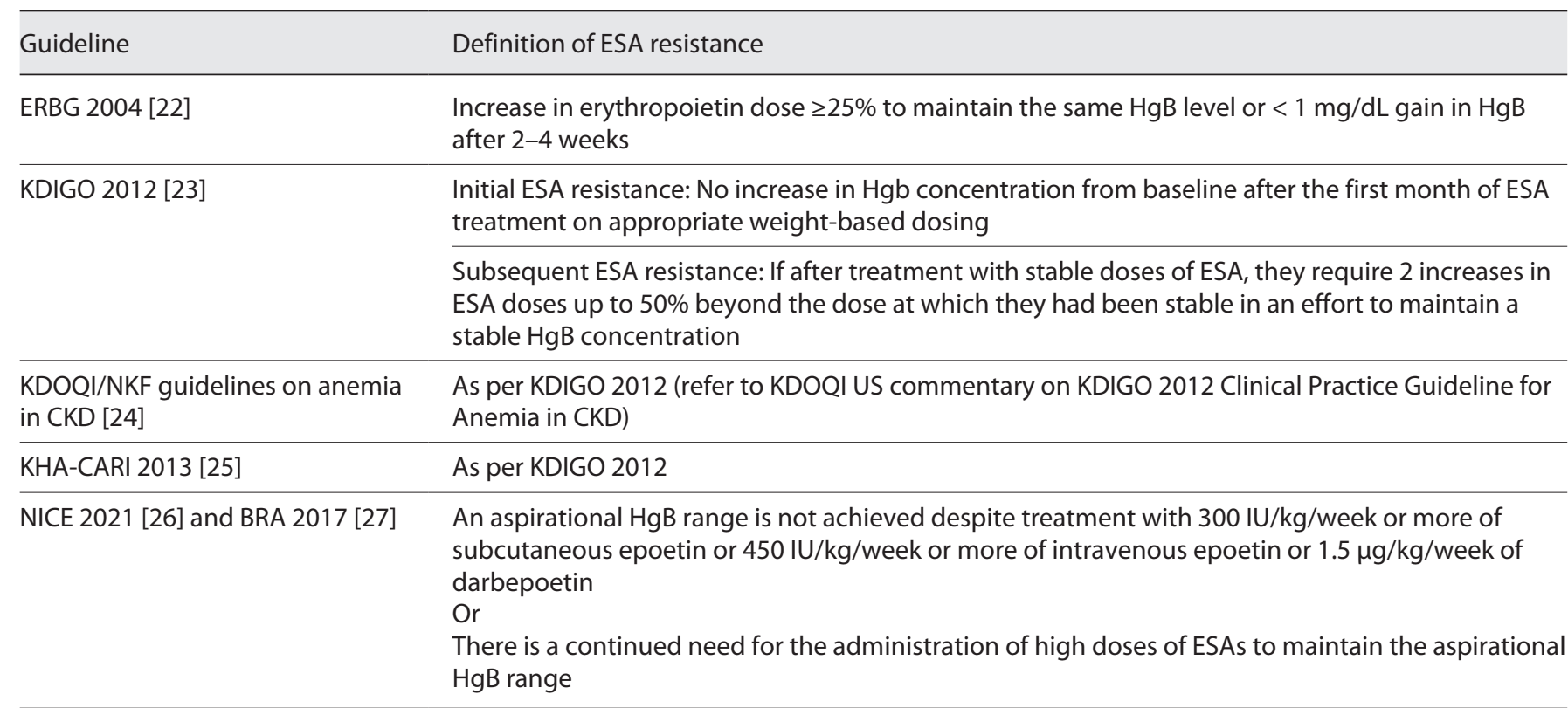

ERBG, European Best Practice Guidelines; KDOQI, Kidney Disease Outcomes Quality Initiative; NKF, National Kidney Foundation; KDIGO, Kidney Disease Improving Global Outcome; KHA-CARI, Kidney Health Australia-Caring for Australasians with Renal Impairment; NICE, National Institute of Clinical Excellence; BRA, British Renal Association; HgB, hemoglobin; CKD, chronic kidney disease; ESA, erythropoietinstimulating agent.

conducted a prospective observational study to evaluate ESA hyporesponsiveness in nondialysis CKD patients over a 4 -year period by dividing ESA responsiveness into good, intermediate, and poor categories, and observed $34 \%$ of the study cohorts were placed in the poor ESA responsiveness group.

Race was noted as a risk factor for ESA unresponsiveness in an observational study on 20,516 patients receiving HD by Okoro et al. [30] though this association is not significant when taking age, gender, and dialysis vintage into account in a multivariate model. Further work is required to evaluate ESA hyporesponsiveness across various health systems internationally and the impact of socioeconomic factors on this.

\section{Clinical Significance of ESA Hyporesponsiveness in CKD}

Over the past decade, clinical outcomes of ESA hyporesponsiveness were investigated with greater frequency. Outcomes in HD patients attracted most research attention $[3,31,32]$. In a study assessing ESA dosing and responsiveness against composite events as defined by car- diovascular events, infection, hospitalization, and mortality, Kuragano et al. [31] have shown that elevated ESA dosage and hyporesponsiveness are correlated to increased occurrence of adverse clinical events, among 1,095 maintenance HD patients followed up over 2 years. The "RISchio CArdiovascolare nei pazienti afferenti all'Area Vasta In Dialis" (RISCAVID) study followed up 753 patients on maintenance HD over a 36 -month period in which demographic, clinical, and laboratory data; comorbidity conditions; administered drugs; all-cause mortality; and fatal and nonfatal cardiovascular events were recorded [33]. The investigators measured ERI, C-reactive protein (CRP), and interleukin-6 (IL-6) in study patients. With ERI values categorized in quartiles (quartile I <5.6, quartile II 5.7-9.6, quartile III 9.7-15.4, and quartile IV >15.4), ERI demonstrated a significant correlation with all-cause mortality as well as fatal and nonfatal cardiovascular events (RR 1.97, 95\% CI 1.39-2.79 and RR 1.62 , 95\% CI 1.12-2.33, respectively) in the RISCAVID study [33]. Furthermore, CRP levels were higher in patients with the highest quartile of ESA hyporesponsiveness, i.e., quartile IV $(p<0.001)$, and predicted all-cause mortality and cardiovascular events [33]. In the RISCAVID study, IL-6 was shown to be a strong predictor of ESA 
Table 2. Potential factors of ESA hyporesponsiveness and responding treatment

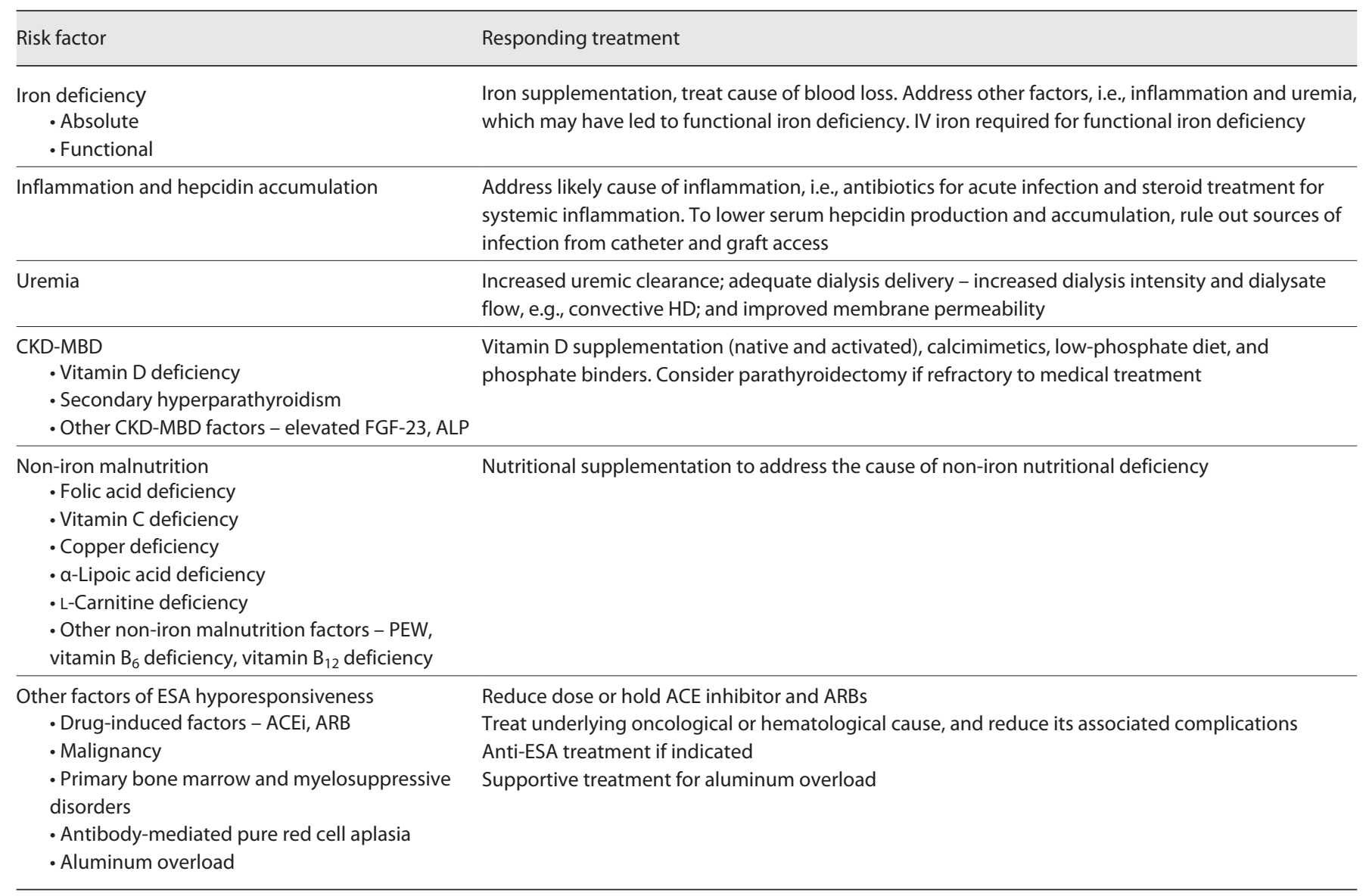

CKD-MBD, chronic kidney disease-mineral bone disease; FGF-23: fibroblast growth factor-23; ALP, alkaline phosphatase; PEW, protein energy wasting; ACEi, angiotensin-converting enzyme inhibitors; ARB, angiotensin receptor blocker; ESA, erythropoietin-stimulating agent; HD, hemodialysis.

hyporesponsiveness; however, this was not the case for CRP [33].

Associations between ESA hyporesponsiveness and first-time thrombotic events are extensively validated in HD patients $[3,32,34]$. Recent data have also highlighted an increased healthcare cost associated with poorer clinical outcomes in ESA hyporesponsiveness [35].

Observational studies discussing adverse outcomes in ESA hyporesponsiveness for non-HD CKD groups remain limited [36]. Hard evidence showing associations between ESA hyporesponsiveness and other outcome parameters, such as renal function trajectory, is scarce. Future aims to address these research gaps should involve a wider population of CKD patients receiving different forms of renal replacement therapy.

\section{Factors Associated with ESA Hyporesponsiveness in CKD}

A summary of currently known factors associated with ESA hyporesponsiveness in CKD and their responding treatments is shown in Table 2.

\section{Iron Deficiency}

Iron deficiency is the most common cause of ESA hyporesponsiveness in $\mathrm{CKD}$, with erythropoiesis being the primary consumer of iron in the human body. Major biomarkers of iron status are serum ferritin and transferrin. Ferritin reflects the body's iron storage, but may not be entirely accurate in an inflammatory state due to its acute phase protein profile. It may be raised in patients with iron deficiency anemia when there is concurrent liver disease or malignancy [37]. Transferrin is a plasma protein 
which transports iron across the bloodstream for utilization and is an indicator of functional iron availability. Transferrin levels fall in iron deficiency [37].

Absolute iron deficiency is defined by a reduced transferrin saturation (TSAT) level of $\leq 20 \%$, and serum ferritin concentration of $\leq 100 \mathrm{ng} / \mathrm{mL}$ for pre-dialysis and peritoneal dialysis patients or $\leq 200 \mathrm{ng} / \mathrm{mL}$ for HD patients $[37,38]$. Blood loss, such as gastrointestinal bleeding and excess menstruation, leads to increased iron demands [39]. Absolute iron deficiency is caused by inadequate gastrointestinal absorption relating to disorders such as inflammatory bowel disease and celiac disease [39]. Inadequate dietary intake is frequently observed in CKD. Poor nutritional balance and chronic alcoholism are also contributing factors toward absolute iron deficiency [40].

Functional iron deficiency is currently defined by ferritin concentration $>100 \mathrm{ng} / \mathrm{mL}$ with TSAT $<20 \%$ [37, 38]. Despite adequate iron stores overall, anemia develops because of inefficient iron utilization [37]. Anemia of chronic disease is the most well-known cause $[37,38]$. Erythrocytes may appear normocytic or microcytic [38]. Inflammation and the role of hepcidin in functional iron deficiency will be discussed in the next subsections.

\section{Inflammation}

Inflammation is an important factor of ESA hyporesponsiveness and associated clinical outcomes [41]. Between 30 and $50 \%$ of HD patients are found to have elevated CRP and IL-6 levels, even in the absence of identifiable sources of inflammation or infection. Inflammation in uremia could be episodic or chronic, but nonetheless associated with elevated all-cause mortality [42]. Causes of inflammation tend to be multifactorial. The stresses of chronic renal impairment are compounded by comorbidities, increased oxidative stress and infection risk, obesity, and genetic and immunological factors [6, 42, 43]. For patients receiving dialysis, dialysis inadequacy accumulates inflammatory cytokines [44]. Catheter and graft access infections add inflammatory risks [45]. The mechanism of how inflammation occurs in CKD likely relates to decreased renal clearance of tumor necrosis factor- $\alpha$, interleukin-1, and IL-6, among other pro-inflammatory cytokines $[6,46]$. Increased production of these cytokines is expected $[6,46]$. The activation of pro-inflammatory cytokines in uremia is mainly triggered by reduced activity of antioxidant enzymes, superoxide dismutase, and glutathione perioxidase [47]. The pathophysiological mechanism of this process is not fully clear and warrants further investigation. What is known is that inflamma- tory cytokines directly inhibit erythropoiesis and promote apoptosis of erythroid precursors $[6,46]$.

\section{Hepcidin}

Hepcidin is a cysteine-rich, 25-amino acid peptide hormone [48]. Its main action is to inhibit iron entry into plasma from the cellular environment $[49,50]$. Hepcidin reduces circulating iron in the human body by binding to ferroportin, a cellular iron transporter which exports absorbed iron from duodenal enterocytes, recycled iron from splenic and hepatic macrophages, and stored iron from hepatocytes to plasma $[49,50]$. Increased serum hepcidin heightens the frequency of hepcidin-ferroportin binding, and therefore greater inhibition of iron efflux $[49,50]$. Direct hepcidin-ferroportin binding triggers endocytosis in both molecules, consequently leading to lysosomal degradation $[49,50]$.

Hepatocytes are the main location of hepcidin production though other cell types such as macrophages and adipocytes also express hepcidin mRNA [51]. Ongoing research continues to explore sources of extrahepatic hepcidin production. Hepcidin synthesis is stimulated by differing plasma transferrin and stored iron within hepatocytes [50]. Hepcidin production is mainly regulated by feedback signals reflecting systemic iron concentration and storage in the human body, as well as the body's erythropoiesis activity and host defense function [50]. When iron levels are abundant, more hepcidin is produced, while hepatocytes produce less hepcidin in iron deficiency [50]. To a lesser extent, hepcidin production is regulated by erythropoietic demands for iron in the body [50]. Hepcidin production is suppressed during active erythropoiesis, allowing higher iron availability for $\mathrm{HgB}$ synthesis [50]. Although not yet comprehensively explained, suppression of hepcidin production occurs in this context through the erythroid factor, a circulating factor produced by bone marrow erythroid precursors [52]. A summary diagram of hepcidin regulation and action in iron homeostasis is shown in Figure 1 [53].

In CKD, 2 major mechanisms - impaired renal clearance and increased inflammatory status - contribute to elevated serum hepcidin levels [52]. For the well-functioning kidney, hepcidin comfortably passes through the glomerular membrane where it is taken up and degraded in the proximal tubule, similarly to other small-sized proteins. Small fractions of filtered hepcidin will pass intact into urine where it is readily detectable [54]. CKD impairs hepcidin clearance and leads to its accumulation in plasma [54]. Though there are suggestions that serum hepcidin may circulate in association with $\alpha 2$-microglobulin, 
Fig. 1. Hepcidin regulation and action in iron homeostasis (Obtained from Locatelli F., Fishbane S., Block G.A., Macdougall I.C. Targeting hypoxia-inducible factors for the treatment of anemia in chronic kidney disease patients. Am J Nephrol. 2017 Mar; 45(3):187-99. Permission obtained from S. Karger AG, Basel).

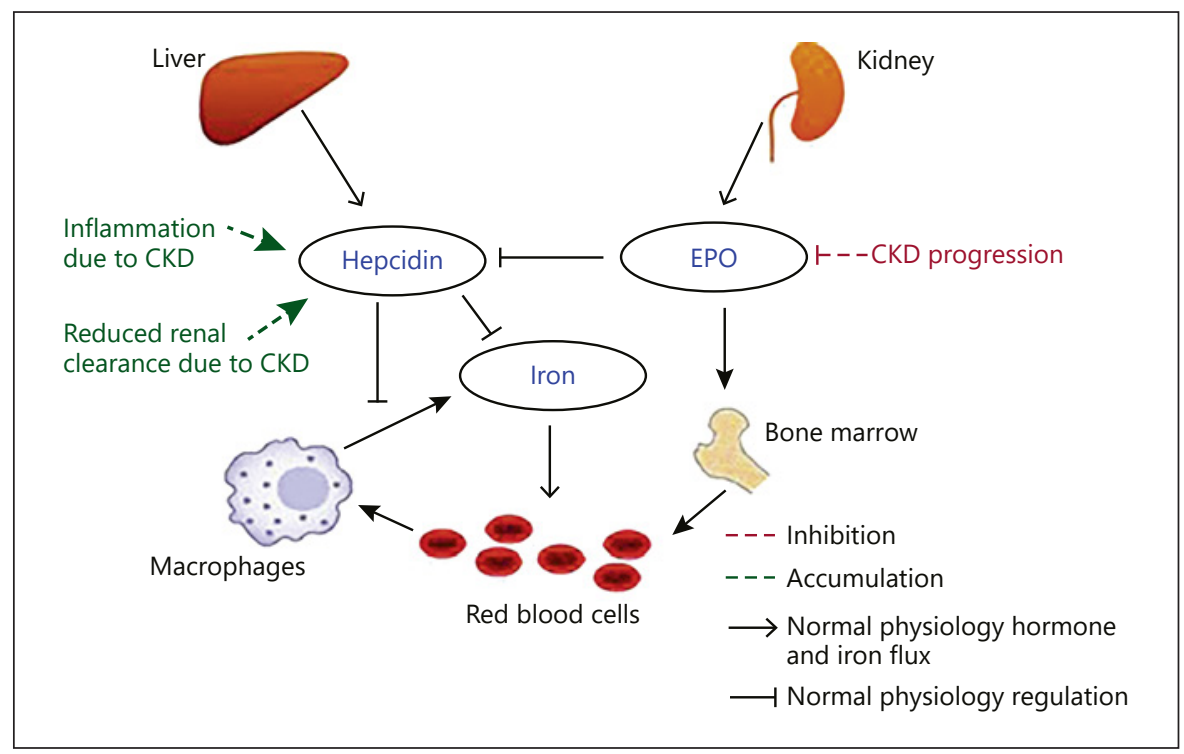

its affinity for hepcidin is low, and a significant proportion of serum hepcidin will be unbounded [55]. Elevated serum hepcidin limits iron availability for erythropoiesis, contributing to ESA hyporesponsiveness.

The transcriptional phase of hepcidin synthesis by hepatocytes is regulated by IL- 6 through the STAT- 3 signaling pathway [56]. Because of this, increased hepcidin production is observed during acute phases of infection and systemic inflammation. Compared to other hepcidin-inducing mechanisms which reduce iron availability, acute infection and inflammation decrease iron levels at the fastest rate [52]. The host defense mechanism involving the multiplication process of iron-dependent extracellular microbes is being limited by the body's functional iron stores [52]. Downside of this mechanism is increased iron sequestration in response. The activation of the reticuloendothelial system and increased hepcidin production will limit the potential of erythropoiesis [52]. Anemia of inflammation (chronic disease) leads to functional iron deficiency and ESA hyporesponsiveness in CKD.

Multiple studies have demonstrated the diagnostic ability of serum hepcidin for ESA hyporesponsiveness in both dialysis and nondialysis CKD patients $[5,6]$. The instantaneous relationship between serum hepcidin levels and ESA hyporesponsiveness still requires further evaluation, and future research is warranted to address this.

\section{CKD-Mineral Bone Disease}

CKD-MBD is a common manifestation in CKD and a recognized factor of ESA hyporesponsiveness. Links among vitamin D deficiency, secondary hyperparathyroidism, and ESA hyporesponsiveness are well-established [57].

The inability to synthesize active vitamin $\mathrm{D}(1,25(\mathrm{OH} 2)$ D3) from its inactive form $25(\mathrm{OH} 2) \mathrm{D} 3$ is the major cause of vitamin D deficiency in CKD [58]. This is exacerbated by inadequate dietary intake, poor sunlight exposure, and nephrotic urinary loss [58]. The pleotropic functions of vitamin $\mathrm{D}$ involve the hematopoietic system, which explains for the role of vitamin D deficiency in ESA hyporesponsiveness $[8,58] .1,25(\mathrm{OH} 2) \mathrm{D} 3$ binds to the vitamin D receptor to exercise its effects on erythropoiesis [8].

Elevated parathyroid hormone (PTH) circulation from secondary hyperparathyroidism is a factor of ESA hyporesponsiveness $[57,59]$. PTH directly inhibits early erythroid progenitors, and reduces endogenous erythropoiesis and red cell survival [59]. Metabolic acidosis and hyperphosphatemia associated with secondary hyperparathyroidism encourage rightward shift of the oxygen$\mathrm{HgB}$ dissociation curve and downregulation of erythropoietin receptors [59]. Excess uremic and inflammatory load exacerbates the hyperparathyroid state through calcium-dependent and calcium-independent mechanisms [59]. Transforming growth factor- $\beta$ and insulin-like growth factor-1 receptor downregulation is exacerbated with secondary hyperparathyroidism in patients with severe uremic states, thereby increasing the burden of ESA hyporesponsiveness [59]. Bone marrow fibrosis, a complication of secondary hyperparathyroidism, has reported associations with ESA hyporesponsiveness [57, 59]. 
In recent years, fibroblast growth factor-23 (FGF-23) and alkaline phosphatase have been advocated as biomarkers to reflect ESA hyporesponsiveness status; however, more extensive evaluation is needed [60, 61]. FGF23 has previously been demonstrated to be a negative regulator of erythropoiesis in mice [60]. Whether iron-restricted erythropoiesis consistently improves with lowering of FGF-23 levels in CKD requires further validation [62].

\section{Non-Iron Malnutrition}

The impact of non-iron nutrition on ESA hyporesponsiveness should not be underestimated. Folic acid is vital to support processes involved in erythroid proliferation, such as nucleotide synthesis, DNA repair, and homocysteine re-methylation [63]. Vitamin $\mathrm{C}$ promotes iron absorption and utilization from tissue stores and downregulates cytokine synthesis from hepatocytes through its role as an antioxidative free oxygen scavenger [64]. Copper encourages iron absorption from the intestinal tract [65]. Both $\alpha$-lipoic acid and L-carnitine play roles to suppress inflammation [66, 67]. $\alpha$-Lipoic acid, required for ATP synthesis, reduces oxidative stress by lowering serum concentration of symmetric-dimethyl arginine [66]. L-Carnitine stimulates heme-oxygenase 1, which has antioxidant effects, and shares the same metabolic pathway with erythropoietin [67]. Protein-energy wasting and vitamin B12 deficiency have previously been shown to display associations with anemia and ESA hyporesponsiveness $[42,68]$. There are conflicting results describing the relationship between vitamin B6 and ESA hyporesponsiveness [69]. The mechanism of these associations requires further investigation.

\section{Other Factors}

Angiotensin-converting enzyme inhibitors and angiotensin-receptor blockers promote ESA hyporesponsiveness through several mechanisms, of which inhibition of angiotensin-II-induced erythropoietin release and augmentation of $\mathrm{N}$-acetyl-seryl-aspartyl-lysyl-proline to prevent recruitment of pluripotent hematopoietic stem cells are the major mechanisms [70]. Patients with cancer, with or without $\mathrm{CKD}$, frequently demonstrate ESA hyporesponsiveness [71]. This is most commonly observed in patients with hematological malignancies such as multiple myeloma and chronic lymphocytic leukemia [71]. ESA hyporesponsiveness has been shown to associate with primary bone marrow disorders and secondary to myelosuppressive agents; however, this is uncommon [72]. Antibody-mediated pure red cell aplasia from the production of neutralizing anti-ESA antibodies during ESA administration is rare [73]. ESA hyporesponsiveness stemming from aluminum overload because of distortion in processes involved in heme synthesis is seldom reported [74].

\section{Management of ESA Hyporesponsiveness and Anemia in CKD}

\section{Iron Supplementation}

There is no clear guidance for an optimal iron supplementation regime in CKD. KDIGO 2012 recommends iron repletion if TSAT $\leq 30 \%$ and serum ferritin $\leq 500 \mathrm{ng} /$ $\mathrm{mL}$ [19]. ERBG 2013 recommended iron repletion only if TSAT $<20 \%$ and serum ferritin $<100 \mathrm{ng} / \mathrm{mL}$, with aims to remain TSAT $\leq 30 \%$ and serum ferritin $\leq 500 \mathrm{ng} / \mathrm{mL}$ [1]. More recent results show high proportions of CKD patients with ferritin $\geq 500 \mathrm{ng} / \mathrm{mL}$ [75]. NICE 2015 and BRA 2017 advised ferritin cutoffs of $800 \mathrm{ng} / \mathrm{mL}$ for patients receiving iron supplementation [22, 23].

Ferrous preparations of orally administered iron are more frequently used because of cost and availability [76]. For CKD patients requiring IV iron, smaller doses and increased frequencies of administration are recommended to improve the cost-effectiveness of iron-restricted erythropoiesis [76].

Ferritin levels in iron supplementation are tightly monitored because of fears regarding iron overload [76]. Concerns relate to damage from oxidative stress, increased risks of infection, atherosclerosis, and tissue-iron deposition [76]. There is increasing evidence to support more liberal regimes of iron supplementation, as demonstrated from results in the PIVOTAL randomized-controlled trial comparing high-dose versus reactive lowdose IV iron supplementation in HD patients [77]. With a ceiling of ferritin $<700 \mathrm{ng} / \mathrm{mL}$ and TSAT $<40 \%$, the high-dose arm demonstrated lower mortality, cardiovascular events, and hospitalization after 2 years of followup, with lower ESA and transfusion requirements [77].

Absolute iron deficiency could be managed with either oral or IV iron, while functional iron deficiency will require IV iron supplementation because of poor intestinal uptake and utilization of iron stores [37, 38]. Numerous trials such as REVOKE and FIND-CKD evaluated the risk-benefit balance for IV iron compared to oral iron use, in which mixed results are seen $[78,79]$.

\section{HIF-Stabilizers}

Since the discovery of the HIF for the EPO gene in 1992, development of drugs, known as HIF-stabilizers or prolyl- 
hydroxylase domain (PHD) inhibitors, acting through the HIF-PHD pathway has brought novel opportunities for renal anemia management. Erythropoiesis in HIF-PHD is dependent on hypoxic status within the cellular environment because oxygen-dependent degradation of HIF controls its levels [16]. In advanced CKD, decreased oxygen diffusion into renal cells occurs because of increased fibrosis, with increased transformation of erythropoietin-producing cells into renal myofibroblasts [80]. Hypoxia is exacerbated by other factors such as destruction of peritubular capillary networks, increased metabolic demands by the renal tubule, and reduced peritubular capillary blood flow [80].

HIF is a heterodimer made up by $\alpha$ and $\beta$ subunits [80]. There are 3 isoforms of the $\alpha$ subunit: HIF- $1 \alpha$, HIF- $2 \alpha$, and HIF- $3 \alpha$ of which all can combine with the $\beta$ subunit to induce expression of different target gene combinations [80]. HIF activity regulation occurs with the HIF- $\alpha$ subunit, synthesized continuously in cells, undergoing hydroxylation at specific proline residues [80]. This process is executed by PHD, and the hydroxylated HIF- $\alpha$ is then ubiquitinated by the von Hippel Lindau-E3 ligase complex before degradation by the proteasome [81] When PHD activity decreases in hypoxia, reduced hydroxylation of HIF- $\alpha$ allows it to stabilize and translocate into the nucleus, where dimerization occurs with HIF- $\beta$ $[80,81]$. Activation of the EPO gene takes place in the nucleus after binding to the hypoxia response element (HRE) at target gene regulatory regions among other genes [80, 81]. In a hypoxic state, increased stabilization of HIF-a will elevate erythropoiesis activity initially [80, 81]. Eventually, HIF function and expression is insufficient to manage demands of erythropoiesis in the hypoxic environment, exacerbated by excess oxidative stress, uremia, and inflammatory cytokine production.

HIF-stabilizers have brought hope to this issue through restoring erythropoiesis in a consistent rate and address factors of ESA hyporesponsiveness. Regulating iron homeostasis to meet iron demands is a key effect. Mechanisms involved in this process increase transferrin, transferrin receptor concentration, duodenal cytochrome B, divalent metal transporter-1, and ceruloplasmin levels [82]. Effects of HIF-stabilizers in suppressing hepcidin and other pro-inflammatory cytokine production have been well-validated $[16,83]$. Associations with improved nutritional status and bone health are observed in trials relating to HIF-stabilizers [83]. Development and integration of HIF-stabilizers into clinical practice has gone from strength to strength with the majority having completed, or in ongoing phase III trials currently [15, 84-87]. They are under close monitoring in the post-market sur- veillance phase throughout the next decade, with approval for clinical use in Asia-Pacific countries such as China and Japan currently [88]. It remains to be seen whether HIF-stabilizers would be approved by the United States Food and Drug Administration, following the unsuccessful application for roxadustat in July 2021.

Although there are multiple advantages of HIF-stabilizers, concerns regarding these drugs are associated with their potential malignancy risks. Transcription of the VEGF gene is regulated by HIF- $1 \alpha$ and HIF- $2 \alpha$ binding to hypoxia response elements [17]. The risk of neoplasia and diabetic retinopathy from HIF-stabilizer use should be recognized as VEGF promotes angiogenesis, vascular permeability, and tumor growth [17]. Phase II studies for vadadustat and daprodustat did not demonstrate change in VEGF levels over the dose range arranged for phase III clinical trials $[89,90]$. Other adverse effects such as metabolic acidosis, hyperkalemia, and upper respiratory tract infections were reported in the world's first HIF-stabilizer phase III clinical trial for roxadustat in the treatment arm $[15,86]$. Going forward, we await more substantive conclusions to determine if HIF-stabilizers is the viable solution to address for ESA hyporesponsiveness in anemia in CKD over long-term.

\section{Management of Inflammatory Factors}

Traditional approach to manage inflammation in CKD is to treat the causative source, whether that is antibiotics for acute infection or steroid treatment for chronic systemic inflammation. Iron supplementation and sufficient dialysis address anemia of inflammation and reduce hepcidin levels. Newer therapies targeted to inhibit hepcidin production are being evaluated. Anti-IL- 6 and IL-6 monoclonal antibody treatment such as tocilizumab and sultuximab are touted as potential options [91, 92]. Atorvastatin use in CKD has been discussed to lower serum hepcidin levels [93]. Recent research also proposed the use of pentoxifylline, a methylxanthine derivative, may have anti-inflammatory effects and improve ESA responsiveness for CKD patients with anemia [94]. Within an optimal concentration range, pentoxifylline suppresses the production of various pro-inflammatory cytokines, such as interleukin-2 and interferon-gamma [94].

\section{Dialysis Adequacy}

Increasing the intensity of dialysis and dialysate flow to reduce ESA hyporesponsiveness could be achieved via numerous mechanisms. Availability of convective HD allows more efficient clearance of middle-weight molecules, such as inflammatory cytokines, and peptides such 


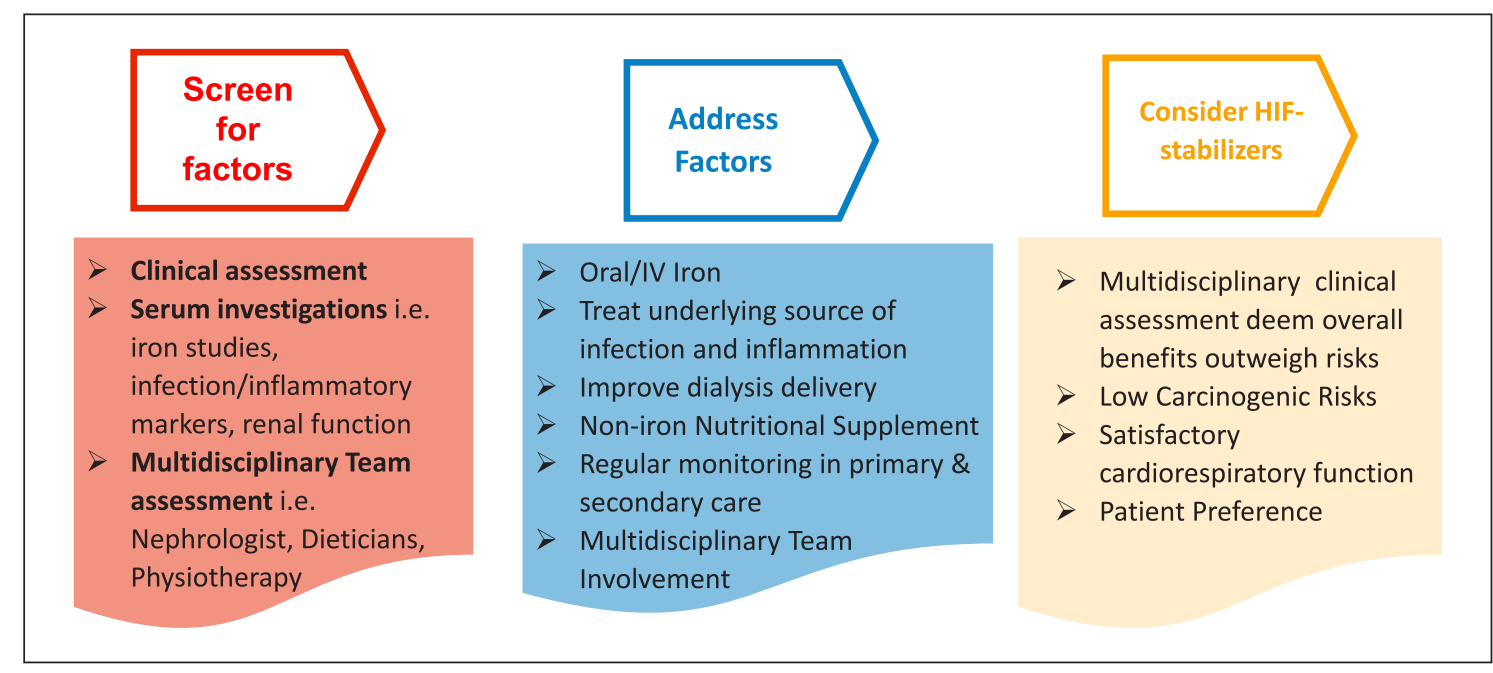

Fig. 2. Proposed pragmatic approach to CKD patients with ESA hyporesponsiveness.

as hepcidin [10]. The REDERT study is a randomized cross-over trial comparing outcomes in patients who underwent high-volume online hemodiafiltration with high-flux polysulfone membranes and exchange volume $20 \mathrm{~L} /$ session versus standard bicarbonate dialysis with low-flux polysulfone membranes [10]. Improved ESA responsiveness and reduced serum hepcidin levels were observed in the online hemodiafiltration group in 3- and 6-month follow-ups, respectively [10].

Options to alter membrane permeability during dialysis have broadened in recent times. Due to antioxidant effects of vitamin E, the application of synthetic vitamin E-bonded dialysis membrane during dialysis has been considered. A multicenter, randomized, controlled trial by Locatelli et al. [11] has illustrated improved ESA responsiveness for patients receiving $\mathrm{HD}$ with vitamin $\mathrm{E}$ coated polysulfone versus a low-flux synthetic dialyzer. Evidence evaluating the impact of membrane permeability on ESA responsiveness remains generally premature, and further validation of these measures is required.

\section{CKD-MBD Management}

Optimizing CKD-MBD treatment may improve ESA responsiveness for patients living with CKD. Vitamin D administered as daily, weekly, or monthly supplements is important as an anti-inflammatory treatment [95]. Use of various vitamin $\mathrm{D}$ analogs and vitamin $\mathrm{D}$ receptor activators has demonstrated increases in ESA responsiveness, particularly for HD groups [96]. Vitamin D supplementation has direct suppressive effects on PTH and serum hepcidin levels [97].

ESA Hyporesponsiveness in CKD
KDIGO 2017 guidelines on CKD-MBD recommend medical management of secondary hyperparathyroidism initially and to consider parathyroidectomy if medical treatment is refractory [98]. Published evidence note improved ESA responsiveness after parathyroidectomy or calcimimetic treatment $[99,100]$. Despite its potential benefits, increased risks of acute mortality from surgical complications should be acknowledged for those undergoing parathyroidectomy [99]. Hyperphosphatemia from secondary hyperparathyroidism is shown to be associated with ESA hyporesponsiveness; however, the mechanism requires more evaluation [101].

\section{Non-Iron Malnutrition Management}

Replenishments to correct non-iron nutrient deficiencies may improve anemia in CKD and ESA responsiveness. Current guidelines do not advocate adjuvant supplementation of these nutrients for nondeficient CKD patients, due to concerns regarding safety levels and risk-benefit balance. An optimal regime for folic acid, vitamin C, copper, $\alpha$-lipoic acid, L-carnitine, and vitamin B6 and B12 supplementation remains desirable. Larger sampled randomized, controlled trials should be conducted to establish this.

\section{Conclusion}

Our understanding of the issues surrounding ESA hyporesponsiveness and potential solutions has certainly expanded over recent years. Despite remaining concerns regarding some of its adverse effects, HIF-stabilizers may 
indeed be the formula to eradicate our concerns over ESA hyporesponsiveness in renal anemia management. Figure 2 illustrates our proposed approach to the assessment and management of ESA hyporesponsiveness based on current evidence. Nonetheless, there are still many gaps in our knowledge base relating to this topic. The nephrology community should endeavor to reach a wider consensus regarding definition, assessment, and management of ESA hyporesponsiveness through continued research efforts to determine a gold-standard pathway. An exciting future awaits to unlock this conundrum.

\section{Conflict of Interest Statement}

The authors have no conflicts of interest to declare.

\section{Funding Sources}

There was no external funding for this work.

\section{Author Contributions}

H.H.L.W. wrote the initial draft. R.C. reviewed the paper and revised it critically. All authors read and approved the final manuscript.

\section{References}

1 Locatelli F, Bárány P, Covic A, De Francisco A, Del Vecchio L, Goldsmith D, et al. ERAEDTA ERBP advisory board. kidney disease: improving global outcomes guidelines on anaemia management in chronic kidney disease: a European renal best practice position statement. Nephrol Dial Transplant. 2013 Jun;28(6):1346-59.

2 United States Renal Data System. Annual data report: atlas of chronic kidney disease and end-stage renal disease in the United States, national institutes of health, national institute of diabetes and digestive and kidney diseases. MD, Bethesda; 2020.

3 Hayashi T, Joki N, Tanaka Y, Iwasaki M, Kubo S, Matsukane A, et al. Resistance to erythropoiesis-stimulating agents in pre-dialysis and post-dialysis mortality in Japanese incident hemodialysis patients. Blood Purif. 2019 Apr;47(2):31-7.

4 Pérez-García R, Varas J, Cives A, Martín-Malo A, Aljama P, Ramos R, et al. Increased mortality in haemodialysis patients administered high doses of erythropoiesis-stimulating agents: a propensity score-matched analysis. Nephrol Dial Transplant. 2018 Apr;33(4): 690-9.

5 Lee SW, Kim JM, Lim HJ, Hwang YH, Kim SW, Chung W, et al. Serum hepcidin may be a novel uremic toxin, which might be related to erythropoietin resistance. Sci Rep. 2017 Jun;7(1):1-8.

6 El Sewefy DA, Farweez BA, Behairy MA, Yassin NR. Impact of serum hepcidin and inflammatory markers on resistance to erythropoiesis-stimulating therapy in haemodialysis patients. Int Urol Nephrol. 2019 Feb;51(2): 325-34.

7 Rosati A, Ravaglia F, Panichi V. Improving erythropoiesis stimulating agent hyporesponsiveness in hemodialysis patients: the role of hepcidin and hemodiafiltration online. Blood Purif. 2018 Apr;45(1-3):139-46.
8 Icardi A, Paoletti E, De Nicola L, Mazzaferro S, Russo R, Cozzolino M. Renal anaemia and EPO hyporesponsiveness associated with vitamin D deficiency: the potential role of inflammation. Nephrol Dial Transplant. 2013 Jul;28(7):1672-9.

9 Fishbane S, Spinowitz B. Update on anemia in ESRD and earlier stages of CKD: core curriculum 2018. Am J Kidney Dis. 2018 Mar;71(3): 423-35.

10 Panichi V, Scatena A, Rosati A, Giusti R, Ferro G, Malagnino E, et al. High-volume online haemodiafiltration improves erythropoiesisstimulating agent (ESA) resistance in comparison with low-flux bicarbonate dialysis: results of the REDERT study. Nephrol Dial Transplant. 2015 Apr;30(4):682-9.

11 Locatelli F, Andrulli S, Viganò SM, Concetti M, Urbini S, Giacchino F, et al. Evaluation of the impact of a new synthetic vitamin Ebonded membrane on the hypo-responsiveness to the erythropoietin therapy in hemodialysis patients: a multicenter study. Blood Purif. 2017 Apr;43(4):338-45.

12 D'Arrigo G, Baggetta R, Tripepi G, Galli F, Bolignano D. Effects of vitamin E-coated versus conventional membranes in chronic hemodialysis patients: a systematic review and meta-analysis. Blood Purif. 2017 Mar;43(13):101-22.

13 Cizman B, Sykes AP, Paul G, Zeig S, Cobitz AR. An exploratory study of daprodustat in erythropoietin-hyporesponsive subjects. Kidney Int Rep. 2018 Jul;3(4):841-50.

14 Haase VH, Chertow GM, Block GA, Pergola PE, deGoma EM, Khawaja Z, et al. Effects of vadadustat on hemoglobin concentrations in patients receiving hemodialysis previously treated with erythropoiesis-stimulating agents. Nephrol Dial Transplant. 2019 Jan; 34(1):90-9.
15 Chen N, Hao C, Liu BC, Lin H, Wang C, Xing $\mathrm{C}$, et al. Roxadustat treatment for anemia in patients undergoing long-term dialysis. $\mathrm{N}$ Engl J Med. 2019 Sep;381(11):1011-22.

16 Nair S, Trivedi M. Anemia management in dialysis patients: a PIVOT and a new path? Curr Opin Nephrol Hypertens. 2020 May;29(3): 351-5.

17 Li Z, Bao S, Wu Q, Wang H, Eyler C, Sathornsumetee $S$, et al. Hypoxia-inducible factors regulate tumorigenic capacity of glioma stem cells. Cancer Cell. 2009 Jun;15(6):501-13.

18 Locatelli F, Aljama P, Bárány P, Canaud B, Carrera F, Eckhardt KU, et al. Cameron $S$ on behalf of the European best practice guidelines working group: revised European best practice guidelines for the management of anaemia in patients with chronic renal failure. Nephrol Dial Transplant. 2004 May;19(2):47.

19 Kidney Disease: Improving Global Outcomes. KDIGO clinical practice guideline for anaemia in chronic kidney disease. Kidney Int Suppl. 2012 Jul;2(4):279-335.

20 National Kidney Foundation. Anaemia. New York; 2021. Available from: https://www.kidney.org/professionals/guidelines/guidelines commentaries/anemia.

21 MacGinley RJ, Walker RG. International treatment guidelines for anaemia in chronic kidney disease: what has changed? Med J Aust. 2013 Jul;199(2):84-5.

22 The National Institute for Health and Care Excellence. NICE Guideline (NG203) chronic kidney disease: managing anaemia. London; 2021 Sep 1. Available from: https://www.nice. org.uk/guidance/ng203.

23 British Renal Association. Clinical practice guideline anemia of chronic kidney disease. Renal Association Clinical Practice Guideline: Anemia of Chronic Kidney Disease. Bristol; 2021 Sep 1. Available from: https:// ukkidney.org/sites/renal.org/files/Updated130220-Anaemia-of-Chronic-Kidney-Disease-1-1.pdf. 
24 Chait Y, Kalim S, Horowitz J, Hollot CV, Ankers ED, Germain MJ, et al. The greatly misunderstood erythropoietin resistance index and the case for a new responsiveness measure. Hemodial Int. 2016 Jul;20(3):392-8.

25 Tsujita M, Kosugi T, Goto N, Futamura K, Nishihira M, Okada M, et al. The effect of maintaining high hemoglobin levels on long-term kidney function in kidney transplant recipients: a randomized controlled trial. Nephrol Dial Transplant. 2019 Aug;34(8):1409-16.

26 Bae MN, Kim SH, Kim YO, Jin DC, Song HC, Choi EJ, et al. Association of erythropoietinstimulating agent responsiveness with mortality in hemodialysis and peritoneal dialysis patients. PloS One. 2015 Nov;10(11):e0143348.

27 Kanbay M, Perazella MA, Kasapoglu B, Koroglu M, Covic A. Erythropoiesis stimulatory agent-resistant anemia in dialysis patients: review of causes and management. Blood Purif. 2010 Jan;29(1):1-2.

28 Ingrasciotta Y, Giorgianni F, Marcianò I, Bolcato J, Pirolo R, Chinellato A, et al. Comparative effectiveness of biosimilar, reference product and other erythropoiesis-stimulating agents (ESAs) still covered by patent in chronic kidney disease and cancer patients: an Italian population-based study. PLoS One. 2016 May;11(5):e0155805.

29 Minutolo R, Conte G, Cianciaruso B, Bellizzi V, Camocardi A, De Paola L, et al. Hyporesponsiveness to erythropoiesis-stimulating agents and renal survival in non-dialysis CKD patients. Nephrol Dial Transplant. 2012 Jul; 27(7):2880-6.

30 Okoro T, Carroll C, Chao J, Laurent SS, Shannon J, Clifton C, et al. SP349 Association of race/ethnicity with erythropoietin stimulating agent use and resistance in hemodialysis patients. Nephrol Dial Transplant. 2019 Jun; 34(1):gfz103-SP349.

31 Kuragano $\mathrm{T}$, Kitamura $\mathrm{K}$, Matsumura $\mathrm{O}$, Matsuda A, Hara T, Kiyomoto H, et al. ESA hyporesponsiveness is associated with adverse events in maintenance hemodialysis (MHD) patients, but not with iron storage. PLoS One. 2016 Mar;11(3):e0147328.

32 Solomon SD, Uno H, Lewis EF, Eckardt KU, Lin J, Burdmann EA, et al. Erythropoietic response and outcomes in kidney disease and type 2 diabetes. New Engl J Med. 2010 Sep; 363(12):1146-55.

33 Panichi V, Rosati A, Bigazzi R, Paoletti S, Mantuano E, Beati S, et al. Anaemia and resistance to erythropoiesis-stimulating agents as prognostic factors in haemodialysis patients: results from the RISCAVID study. Nephrol Dial Transplant. 2011 Aug;26(8):2641-8.

34 Corwin HL, Gettinger A, Fabian TC, May A, Pearl RG, Heard S, et al. Efficacy and safety of epoetin alfa in critically ill patients. New Engl J Med. 2007 Sep;357(10):965-76.

35 Cizman B, Smith HT, Camejo RR, Casillas L, Dhillon $\mathrm{H}, \mathrm{Mu} \mathrm{F}$, et al. Clinical and economic outcomes of erythropoiesis-stimulating agent hyporesponsiveness in the post-bundling era. Kidney Med. 2020 Sep;2(5):589-99.
36 Guedes M, Robinson BM, Obrador G, Tong A, Pisoni RL, Pecoits-Filho R. Management of anemia in non-dialysis chronic kidney disease: current recommendations, real-world practice, and patient perspectives. Kidney 360 . 2020 Jan;1(1):855-62.

37 Wish JB. Assessing iron status: beyond serum ferritin and transferrin saturation. Clin J Am Soc Nephrol. 2006 Sep;1(1):S4-8.

38 NKF Anemia Working Group. Clinical practice guidelines for anemia of chronic kidney disease: update 2000. Am J Kidney Dis. 2001 Jan;37(Suppl 1):S182-238.

39 Camaschella C. Iron-deficiency anemia. N Engl J Med. 2015 May;372(19):1832-43.

40 Lindenbaum J, Roman MJ. Nutritional anemia in alcoholism. Am J Clin Nutr. 1980 Dec; 33(12):2727-35.

41 Karaboyas A, Morgenstern H, Fleischer NL, Vanholder RC, Dhalwani NN, Schaeffner E, et al. Inflammation and erythropoiesis-stimulating agent response in hemodialysis patients: a self-matched longitudinal study of anemia management in the dialysis outcomes and practice patterns study (DOPPS). Kidney Med. 2020 May;2(3):286-96.

42 Jofré R, Rodriguez-Benitez P, López-Gómez JM, Pérez-Garcia R. Inflammatory syndrome in patients on hemodialysis. J Am Soc Nephrol. 2006 Dec;17(12):S274-80.

43 Kalantar-Zadeh K, Ikizler TA, Block G, Avram MM, Kopple JD. Malnutrition-inflammation complex syndrome in dialysis patients: causes and consequences. Am J Kidney Dis. 2003 Nov; 42(5):864-81.

44 Schulman G. Poor nutritional status and inflammation: the dose of dialysis in hemodialysis patients: impact on nutrition. Semin Dial. 2004 Nov; 17(6):479-88.

45 Nassar GM, Ayus JC. Infectious complications of the hemodialysis access. Kidney Int. $2001 \mathrm{Jul} ; 60(1): 1-13$.

46 Macdougall IC, Cooper AC. Erythropoietin resistance: the role of inflammation and proinflammatory cytokines. Nephrol Dial Transplant. 2002 Nov; 17(11):39-43.

47 Xu G, Luo K, Liu H, Huang T, Fang X, Tu W. The progress of inflammation and oxidative stress in patients with chronic kidney disease. Ren Fail. 2015 Jan;37(1):45-9.

48 Park $\mathrm{CH}$, Valore EV, Waring AJ, Ganz T. Hepcidin, a urinary antimicrobial peptide synthesized in the liver. J Biol Chem. 2001 Mar;276(11):7806-10.

49 Nemeth E, Tuttle MS, Powelson J, Vaughn MB, Donovan A, Ward DM, et al. Hepcidin regulates cellular iron efflux by binding to ferroportin and inducing its internalization. Science. 2004 Dec;306(5704):2090-3.

50 De Domenico I, Ward DM, Kaplan J. Hepcidin regulation: ironing out the details. J Clin Invest. $2007 \mathrm{Jul} ; 117(7): 1755-8$.

51 Liu XB, Nguyen NB, Marquess KD, Yang F, Haile DJ. Regulation of hepcidin and ferroportin expression by lipopolysaccharide in splenic macrophages. Blood Cells Mol Dis. 2005 Jul;35(1):47-56.
52 Ganz T, Nemeth E. Hepcidin and iron homeostasis. Biochim Biophys Acta. 2012 Sep; 1823(9):1434-43.

53 Locatelli F, Fishbane S, Block GA, Macdougall IC. Targeting hypoxia-inducible factors for the treatment of anemia in chronic kidney disease patients. Am J Nephrol. 2017 Mar; 45(3):187-99.

54 Ganz T, Olbina G, Girelli D, Nemeth E, Westerman M. Immunoassay for human serum hepcidin. Blood. 2008 Nov;112(10):4292-7.

55 Peslova G, Petrak J, Kuzelova K, Hrdy I, Halada P, Kuchel PW, et al. Hepcidin, the hormone of iron metabolism, is bound specifically to $\alpha$-2-macroglobulin in blood. Blood. 2009 Jun;113(24):6225-36.

56 Wrighting DM, Andrews NC. Interleukin-6 induces hepcidin expression through STAT3. Blood. 2006 Nov; 108(9):3204-9.

57 Bamgbola OF. Pattern of resistance to erythropoietin-stimulating agents in chronic kidney disease. Kidney Int. 2011 Sep;80(5):464-74.

58 Koenig KG, Lindberg JS, Zerwekh JE, Padalino PK, Cushner HM, Copley JB. Free and total 1,25-dihydroxyvitamin $\mathrm{D}$ levels in subjects with renal disease. Kidney Int. 1992 Jan;41(1): 161-5.

59 Del Vecchio L, Pozzoni P, Andrulli S, Locatelli F. Inflammation and resistance to treatment with recombinant human erythropoietin. J Ren Nutr. 2005 Jan;15(1):137-41.

60 Coe LM, Madathil SV, Casu C, Lanske B, Rivella S, Sitara D. FGF-23 is a negative regulator of prenatal and postnatal erythropoiesis. J Biol Chem. 2014 Apr;289(14):9795-810.

61 Usui T, Zhao J, Fuller DS, Hanafusa N, Hasegawa T, Fujino H, et al. Association of erythropoietin resistance and fibroblast growth factor 23 in dialysis patients: results from the Japanese dialysis outcomes and practice patterns study. Nephrology. 2021 Jan;26(1):46-53.

62 Maruyama N, Otsuki T, Yoshida Y, Nagura C, Kitai M, Shibahara N, et al. Ferric citrate decreases fibroblast growth factor 23 and improves erythropoietin responsiveness in hemodialysis patients. Am J Nephrol. 2018 Jul; 47(6):406-14.

63 Bamonti-Catena F, Buccianti G, Porcella A, Valenti G, Como G, Finazzi S, et al. Folate measurements in patients on regular hemodialysis treatment. Am J Kidney Dis. 1999 Mar; 33(3):492-7.

64 Bridges KR, Hoffman KE. The effects of ascorbic acid on the intracellular metabolism of iron and ferritin. J Biol Chem. 1986 Oct; 261(30):14273-7.

65 Ikee R, Tsunoda M, Sasaki N, Sato N, Hashimoto N. Clinical factors associated with serum copper levels and potential effect of sevelamer in hemodialysis patients. Int Urol Nephrol. 2013 Jun;45(3):839-45.

66 Tan BL, Norhaizan ME, Liew WP. Nutrients and oxidative stress: friend or foe? Oxid Med Cell Longev. 2018 Jan;2018:9719584. 
67 Calò LA, Davis PA, Pagnin E, Bertipaglia L, Naso A, Piccoli A, et al. Carnitine-mediated improved response to erythropoietin involves induction of haem oxygenase-1: studies in humans and in an animal model. Nephrol Dial Transplant. 2008 Mar;23(3):890-5.

68 Killen JP, Brenninger VL. Hydroxocobalamin supplementation and erythropoisis stimulating agent hyporesponsiveness in haemodialysis patients. Nephrology. 2014 Mar;19(3): 164-71.

69 Obi Y, Mikami S, Hamano T, Obi Y, Tanaka $\mathrm{H}$, Shimomura A, et al. Intravenous Vitamin B6 increases resistance to erythropoiesisstimulating agents in hemodialysis patients: a randomized controlled trial. J Ren Nutr. 2016 Nov;26(6):380-90.

70 Azizi M, Rousseau A, Ezan E, Guyene TT, Michelet S, Grognet JM, et al. Acute angiotensinconverting enzyme inhibition increases the plasma level of the natural stem cell regulator $\mathrm{N}$-acetyl-seryl-aspartyl-lysyl-proline. J Clin Invest. $1996 \mathrm{Feb}$;97(3):839-44.

71 Latcha S. Anemia management in cancer patients with chronic kidney disease. Semin Dial. 2019 Nov;32(6):513-9.

72 Drüeke T. Hyporesponsiveness to recombinant human erythropoietin. Nephrol Dial Transplant. 2001 Oct;16(7):25-8.

73 Del Vecchio L, Locatelli F. An overview on safety issues related to erythropoiesis-stimulating agents for the treatment of anaemia in patients with chronic kidney disease. Expert Opin Drug Saf. 2016 Aug;15(8):1021-30.

74 Casadevall N. Cellular mechanism of resistance to erythropoietin. Nephrol Dial Transplant. 1995 Jan;10(Suppl 6):27-30.

75 Fuller DS, Pisoni RL, Bieber BA, Port FK, Robinson BM. The DOPPS practice monitor for US dialysis care: update on trends in anemia management 2 years into the bundle. Am J Kidney Dis. 2013 Dec;62(6):1213-6.

76 Del Vecchio L, Longhi S, Locatelli F. Safety concerns about intravenous iron therapy in patients with chronic kidney disease. Clin Kidney J. 2016 Apr;9(2):260-7.

77 Macdougall IC, White C, Anker SD, Bhandari S, Farrington K, Kalra PA, et al. Intravenous iron in patients undergoing maintenance hemodialysis. N Engl J Med. 2019 Jan;380(5): $447-58$.

78 Agarwal R, Kusek JW, Pappas MK. A randomized trial of intravenous and oral iron in chronic kidney disease. Kidney Int. 2015 Oct; 88(4):905-14.

79 Macdougall IC, Bock AH, Carrera F, Eckardt KU, Gaillard C, Van Wyck D, et al. FINDCKD: a randomized trial of intravenous ferric carboxymaltose versus oral iron in patients with chronic kidney disease and iron deficiency anaemia. Nephrol Dial Transplant. 2014 Nov;29(11):2075-84.
80 Rosenberger C, Mandriota S, Jürgensen JS, Wiesener MS, Hörstrup JH, Frei U, et al. Expression of hypoxia-inducible factor- $1 \alpha$ and$2 \alpha$ in hypoxic and ischemic rat kidneys. J Am Soc Nephrol. 2002 Jul;13(7):1721-32.

81 Jaakkola P, Mole DR, Tian YM, Wilson MI, Gielbert J, Gaskell SJ, et al. Targeting of HIF-a to the von Hippel-Lindau ubiquitylation complex by $\mathrm{O} 2$-regulated prolyl hydroxylation. Science. 2001 Apr;292(5516):468-72.

82 Kular D, Macdougall IC. HIF stabilizers in the management of renal anemia: from bench to bedside to pediatrics. Pediatr Nephrol. 2019 Mar;34(3):365-78.

83 Panwar B, Gutiérrez OM. Disorders of iron metabolism and anemia in chronic kidney disease. Semin Nephrol. 2016 Jul;36(4):25261.

84 Akizawa T, Nangaku M, Yonekawa T, Okuda N, Kawamatsu S, Onoue T, et al. Efficacy and safety of daprodustat compared with darbepoetin alfa in Japanese hemodialysis patients with anemia: a randomized, double-blind, phase 3 trial. Clin J Am Soc Nephrol. 2020 Aug; 15(8):1155-65.

85 Eckardt KU, Agarwal R, Farag YM, Jardine AG, Khawaja Z, Koury MJ, et al. Global Phase 3 programme of vadadustat for treatment of anaemia of chronic kidney disease: rationale, study design and baseline characteristics of dialysis-dependent patients in the INNO2VATE trials. Nephrol Dial Transplant. 2020.

86 Chen N, Hao C, Peng X, Lin H, Yin A, Hao L, et al. Roxadustat for anemia in patients with kidney disease not receiving dialysis. New Engl J Med. 2019 Sep;381(11):1001-10.

87 Macdougall IC, Akizawa T, Berns JS, Bernhardt T, Krueger T. Effects of molidustat in the treatment of anemia in CKD. Clin J Am Soc Nephrol. 2019 Jan;14(1):28-39.

88 Yap DY, McMahon LP, Hao CM, Hu N, Okada H, Suzuki Y, et al. Recommendations by the Asian pacific society of nephrology (APSN) on the appropriate use of HIF-PH inhibitors. Nephrology. 2021 Feb;26(2):105-18.

89 Yousaf F, Spinowitz B. Hypoxia-inducible factor stabilizers: a new avenue for reducing $\mathrm{BP}$ while helping hemoglobin? Curr Hypertens Rep. 2016 Mar; 18(3):23.

90 Seeley TW, Sternlicht MD, Klaus SJ, Neff TB, Liu DY. Induction of erythropoiesis by hypoxia-inducible factor prolyl hydroxylase inhibitors without promotion of tumor initiation, progression, or metastasis in a VEGFsensitive model of spontaneous breast cancer. Hypoxia. 2017 Mar;5:1-9.

91 Song SN, Tomosugi N, Kawabata H, Ishikawa T, Nishikawa T, Yoshizaki K. Down-regulation of hepcidin resulting from long-term treatment with an anti-IL-6 receptor antibody (tocilizumab) improves anemia of inflammation in multicentric Castleman disease. Blood. 2010 Nov;116(18):3627-34
92 Casper C, Chaturvedi S, Munshi N, Wong R, Qi M, Schaffer M, et al. Analysis of inflammatory and anemia-related biomarkers in a randomized, double-blind, placebo-controlled study of siltuximab (anti-IL6 monoclonal antibody) in patients with multicentric Castleman disease. Clin Cancer Res. 2015 Oct;21(19):4294-304

93 Masajtis-Zagajewska A, Nowicki M. Effect of atorvastatin on iron metabolism regulation in patients with chronic kidney disease: a randomized double blind crossover study. Ren Fail. 2018 Oct;40(1):700-9.

94 Johnson DW, Pascoe EM, Badve SV, Dalziel $\mathrm{K}$, Cass A, Clarke P, et al. A randomized, placebo-controlled trial of pentoxifylline on erythropoiesis-stimulating agent hyporesponsiveness in anemic patients with CKD: the handling erythropoietin resistance with oxpentifylline (HERO) trial. Am J Kidney Dis. 2015 Jan;65(1):49-57.

95 Yin K, Agrawal DK. Vitamin D and inflammatory diseases. J Inflamm Res. 2014 May;7: 69-87.

96 Moorthi RN, Kandula P, Moe SM. Optimal vitamin $\mathrm{D}$, calcitriol, and vitamin $\mathrm{D}$ analog replacement in chronic kidney disease: to $\mathrm{D}$ or not to D: that is the question. Curr Opin Nephrol Hypertens. 2011 Jul;20(4):354-9.

97 Bacchetta J, Zaritsky JJ, Sea JL, Chun RF, Lisse TS, Zavala K, et al. Suppression of ironregulatory hepcidin by vitamin D. J Am Soc Nephrol. 2014 Mar;25(3):564-72.

98 Ketteler M, Block GA, Evenepoel P, Fukagawa M, Herzog CA, McCann L, et al. Executive summary of the 2017 KDIGO chronic kidney disease - mineral and bone disorder (CKD-MBD) guideline update: what's changed and why it matters. Kidney Int. 2017 Jul;92(1):26-36.

99 Trunzo JA, McHenry CR, Schulak JA, Wilhelm SM. Effect of parathyroidectomy on anemia and erythropoietin dosing in endstage renal disease patients with hyperparathyroidism. Surgery. 2008 Dec;144(6):915-

100 Tanaka M, Yoshida K, Fukuma S, Ito K, Matsushita K, Fukagawa M, et al. Effects of secondary hyperparathyroidism treatment on improvement in anemia: results from the MBD-5D study. PLoS One. 2016 Oct;11(10): e0164865.

101 Kuwahara M, Mandai S, Kasagi Y, Kusaka K, Tanaka T, Shikuma S, et al. Responsiveness to erythropoiesis-stimulating agents and renal survival in patients with chronic kidney disease. Clin Exp Nephrol. 2015 Aug;19(4): 598-605. 